\title{
Structure of GaN(0001): The laterally contracted Ga bilayer model
}

\author{
John E. Northrup \\ Xerox Palo Alto Research Center, 3333 Coyote Hill Road, Palo Alto, California 94304 \\ J. Neugebauer \\ Fritz-Haber-Institut der MPG, Faradeyweg 4-6, D-13195 Berlin, Germany \\ R. M. Feenstra \\ Physics Department, Carnegie Mellon University, Pittsburgh, Pennsylvania 15213
}

\author{
A. R. Smith \\ Physics Department, Ohio University, Athens, Ohio 45701
}

(Received 23 November 1999)

\begin{abstract}
We discuss the energetics and structure of a laterally contracted Ga bilayer model for the Ga-rich pseudo$1 \times 1$ phase of the $\mathrm{GaN}(0001)$ surface. First-principles total energy calculations reveal that a laterally contracted overlayer of $\mathrm{Ga}$ atoms bonded to a $1 \times 1 \mathrm{Ga}$ adlayer is energetically favorable in the Ga-rich limit. The calculations also show that the energy of this surface structure is very insensitive to the lateral position of the contracted layer with respect to the underlying Ga layer. The flatness of the energy surface suggests the presence of rapidly moving domain boundaries separating regions of the surface having different registries. Such motion may lead to the $1 \times 1$ corrugation pattern seen in scanning tunneling microscopy images.
\end{abstract}

Because of its importance as a material enabling the fabrication of optoelectronic and electronic devices, $\mathrm{GaN}$ has been studied extensively in recent years. In most cases, GaN films must be obtained by epitaxial growth on a foreign substrate such as $\mathrm{Al}_{2} \mathrm{O}_{3}(0001)$ or $\mathrm{SiC}(0001)$. Because the highest quality material is generally grown on the (0001) plane of $\mathrm{GaN}$, the structure of the (0001) surface has been studied both experimentally and theoretically. Part of the motivation underlying these efforts to identify the surface atomic structure stems from the impact such knowledge would have on attempts to understand growth morphology and dopant incorporation. Both of these phenomena are influenced by surface atomic structure and its effect on adatom diffusion and incorporation kinetics.

The symmetry and local atomic structure of the reconstructions that are obtained during and after molecular-beam epitaxy (MBE) growth on the $\mathrm{GaN}(0001)$ surface have been subjects of a number of recent investigations. ${ }^{1-10}$ As the Ga concentration on the surface increases, the symmetry of the reconstructions changes from $2 \times 2$ to $5 \times 5$ to $4 \times 6$ and finally to the structure that has been termed the pseudo- $1 \times 1$ structure. ${ }^{1,6,9}$ The pseudo- $1 \times 1$ phase has been obtained experimentally by terminating MBE growth under Ga-rich conditions and is probably very similar to the structure prevailing during Ga-rich growth.

One of the fascinating aspects of the pseudo- $1 \times 1$ surface is the observation by scanning tunneling microscopy (STM) of a $1 \times 1$ corrugation pattern that has the same lateral spacing as bulk GaN. ${ }^{1}$ At the same time, however, electron diffraction experiments performed at temperatures less than $350{ }^{\circ} \mathrm{C}$ reveal additional diffraction intensity in satellite spots, indicating that the actual lattice vectors of the surface unit cell are much larger than those corresponding to a 1 $\times 1$ cell. Modeling of the Auger spectral intensities indicates that the pseudo- $1 \times 1$ contains between 2 and $3 \mathrm{ML}$ of $\mathrm{Ga}$ above the last $\mathrm{GaN}$ bilayer. On the basis of these observations, as well as energy considerations, it has been proposed ${ }^{1}$ that the pseudo- $1 \times 1$ surface is an incommensurate laterally contracted bilayer structure, and that the $1 \times 1$ corrugation pattern seen in the STM is a result of the time averaging inherent in the STM measurement. It was suggested that the surface Ga atoms are sufficiently ordered to give rise to the satellite features in electron diffraction, yet sufficiently mobile so that the STM image reflects the time-average vertical corrugation produced by the potential energy surface seen by the $\mathrm{Ga}$ atoms as they move over the $1 \times 1$ substrate. At room temperature, the motion of the Ga may be facilitated by mobile point defects or mobile domain boundaries separating regions that have different registrations of the $\mathrm{Ga}$ adlayers with respect to a $1 \times 1$ substrate. ${ }^{1}$ At temperatures greater than $350{ }^{\circ} \mathrm{C}$ these $\mathrm{Ga}$ layers are likely to be fluid. In this paper we present first-principles total energy calculations, which indicate that a surface consisting of a laterally contracted bilayer of $\mathrm{Ga}$ is (under Ga-rich conditions) the most stable structure that has been obtained to date. We find that the energy barrier to lateral translation of the top Ga layer over the underlying layers is on the order of $0.02 \mathrm{eV} / 1 \times 1$. These results provide clear theoretical evidence in favor of a mobile laterally contracted bilayer structure containing $\sim 2.3$ ML of $\mathrm{Ga}$ above the Ga-terminated GaN bilayer. The stability of such an extremely Ga-rich structure has implications for understanding dopant incorporation, surface diffusion, and growth of $\mathrm{GaN}$ under Ga-rich conditions.

Before proceeding to the discussion of the pseudo- $1 \times 1$ surface, we briefly review what is known about the other structures appearing on the (0001) surface. Under N-rich conditions a $2 \times 2$ reconstruction is observed following growth interruptions. ${ }^{2,7-9}$ The available total energy calculations $^{3-5,9}$ for the $\mathrm{GaN}(0001)$ surface suggest that this $2 \times 2$ structure could correspond to either the $\mathrm{N}$ adatom or 
the Ga adatom model as discussed elsewhere. ${ }^{9}$ Because the $2 \times 2$ structure is usually obtained by interrupting the growth by turning off the $\mathrm{Ga}$ flux, but with an activated $\mathrm{N}$ flux still incident on the surface, the $2 \times 2$ local ordering is most likely a consequence of $\mathrm{N}$ adatoms. It has also been shown that one may obtain a $2 \times 2$ structure during growth when As is present on the surface. ${ }^{10}$ On the basis of STM studies it has been suggested that the $5 \times 5$ structure could arise from an ordered mixture of $\mathrm{Ga}$ and $\mathrm{N}$ adatoms. ${ }^{9}$ However, a conclusive determination of the atomic structure of the $5 \times 5$ has not yet been obtained.

With regard to the pseudo- $1 \times 1$ structure, the issue that theory must confront is the identification of a structure that is both consistent with experimental observations and stable with respect to other possible reconstructions, e.g., the $\mathrm{Ga}$ adatom model. Previous work has indicated that structures with 1 or $2 \mathrm{ML}$ of excess Ga, and which have $1 \times 1$ translation symmetry, are not as stable as the $2 \times 2$ Ga-adatom model under Ga-rich conditions. ${ }^{1}$ It was noted in these studies however, that these $1 \times 1 \mathrm{Ga}$ adlayers would be under considerable tensile stress, and would be expected to contract. ${ }^{1}$ In this paper, we present explicit calculations for a laterally contracted bilayer of $\mathrm{Ga}$ and show that such a structure is more stable than the $2 \times 2 \mathrm{Ga}$ adatom model by at least $0.25 \mathrm{eV} / 1 \times 1$ in the Ga-rich limit.

The total energy calculations are performed using the plane-wave pseudopotential method and the local-density approximation. The plane wave cutoff employed in the calculations is $60 \mathrm{Ry}$ and the $\mathrm{Ga} 3 d$ orbitals are included in the valence band. These methods ${ }^{11}$ have been employed in previous calculations for AIN (Ref. 12) and GaN (Refs. 3 and 13) surfaces. We consider cells containing 8 layers of $\mathrm{GaN}$ plus 1 or 2 additional layers of $\mathrm{Ga}$ above these layers. The $\mathrm{N}$ dangling bonds on the backside of the supercell are saturated by pseudohydrogen atoms with charge $\frac{3}{4}$. To determine the relative energies of the structures we employ the thermodynamic formalism ${ }^{14}$ wherein the difference in energies between structures depends linearly on the Ga chemical potential as follows:

$$
\Delta E=\Delta E(\text { Ga-rich })+\left(\Delta n_{\mathrm{N}}-\Delta n_{\mathrm{Ga}}\right)\left(\mu_{\mathrm{Ga}}-\mu_{\mathrm{Ga}(\text { bulk })}\right) .
$$

In this expression $\Delta n_{\mathrm{N}}$ and $\Delta n_{\mathrm{Ga}}$ are the differences, per unit area, in the numbers of $\mathrm{N}$ and $\mathrm{Ga}$ atoms in the structures, $\mu_{\mathrm{Ga}}$ is the chemical potential of $\mathrm{Ga}$, and $\mu_{(\mathrm{Ga} \text { (bulk)) }}$ is the energy per atom of bulk Ga. The maximum allowable value for $\mu_{\mathrm{Ga}}$ is $\mu_{(\mathrm{Ga} \text { (bulk)) }}$. Thus, by definition we have $\Delta E$ $=\Delta E($ Ga-rich $)$ in the Ga-rich limit.

To model a laterally contracted adlayer structure we employ a $\sqrt{3} \times \sqrt{3}$ cell. The use of such a cell allows us to model hexagonal $\mathrm{Ga}$ adlayers with a reduced Ga-Ga spacing of $(\sqrt{3} / 2) a_{1 \times 1}$, where $a_{1 \times 1}=3.17 \AA$ is the in-plane spacing of Ga atoms on the $1 \times 1$ ideal surface. In these $\sqrt{3} \times \sqrt{3}$ cells there are four atoms in the laterally contracted hexagonal overlayer for every three atoms in the underlying hexagonal layers. The lattice vectors of the overlayer are rotated by 30 degrees with respect to those of the substrate as shown in Fig. 1. The lateral spacing of the $\mathrm{Ga}$ atoms in the overlayer is contracted to $a_{c}=(\sqrt{3} / 2) a_{1 \times 1}=2.75 \AA$. As will be discussed further below, this Ga-Ga spacing is close to the value which minimizes the formation energy of a free standing layer of

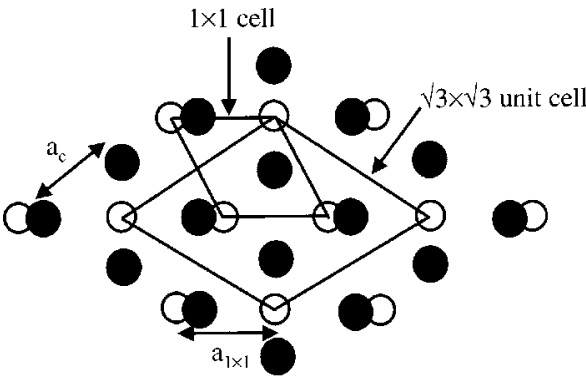

1 1 hexagonal substrate layer
Laterally contracted hexagonal overlayer

FIG. 1. This figure is a schematic top view showing a laterally contracted hexagonal overlayer (filled circles) above a hexagonal substrate layer (open circles). The spacing of the atoms in the overlayer is $a_{c}=(\sqrt{3} / 2) a_{1 \times 1}$. The spacing of the atoms in the substrate is $a_{1 \times 1}$. For GaN, $a_{1 \times 1}=3.17 \AA$. The lattice vectors of the contracted $1 \times 1$ cell of the overlayer are rotated by $30^{\circ}$ with respect to the lattice vectors of the $1 \times 1$ cell of the substrate.

Ga. Consequently, this $\sqrt{3} \times \sqrt{3}$ cell should provide a very good upper bound for the energy of the optimal laterally contracted overlayer structure.

Total energy calculations were performed for laterally contracted adlayer structures containing one or two layers of Ga. These structures are illustrated in Figs. 2 and 3. Shown

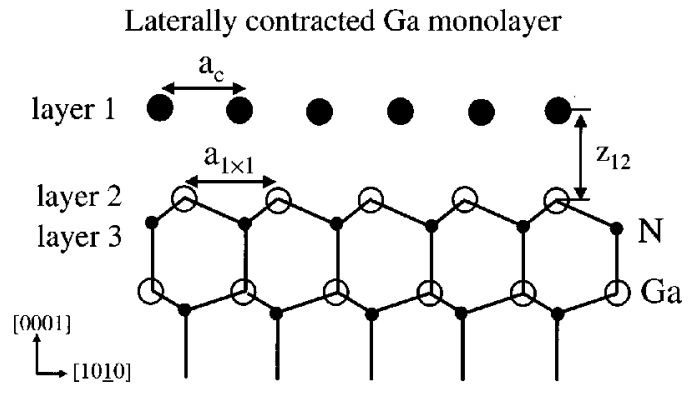

(a)

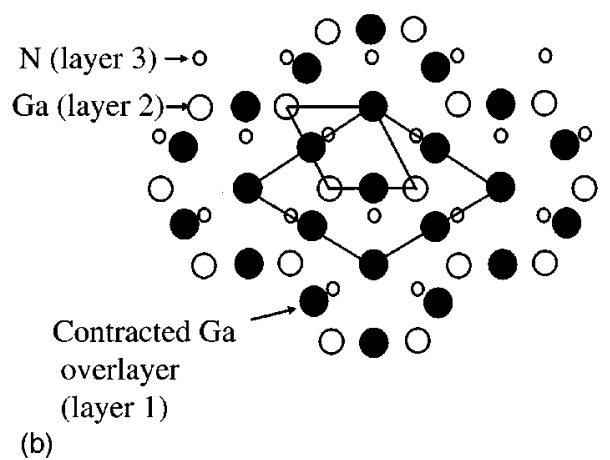

FIG. 2. A laterally contracted monolayer structure is depicted in two projections. (a) A schematic projection of a laterally contracted monolayer (layer 1) above a Ga-terminated (0001) surface. The average separation between layers 1 and 2 is $z_{12}=2.47 \AA$. Note: In this projection the laterally contracted monolayer (layer 1 ) has been rotated by 30 degrees for ease of viewing. (b) A projection of the top three layers of a laterally contracted monolayer structure on the $c$-plane. In the registration depicted here the four atoms in layer 1 occupy one top site $(T 1)$ and three bridge sites. The atoms in the top sites are higher by $0.02 \AA$. 
(a) Laterally contracted bilayer model (time average)

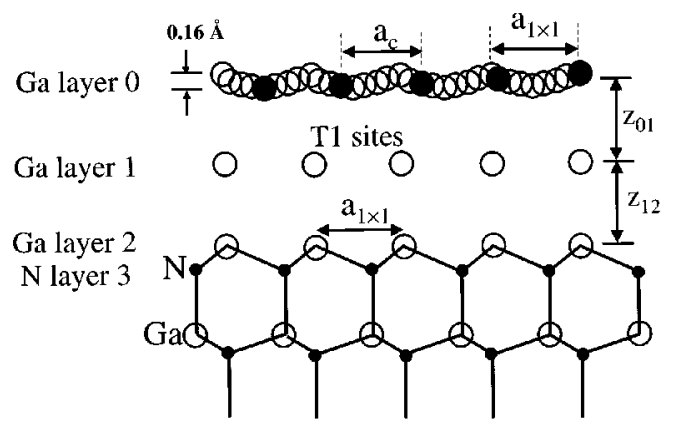

(b) registry $\mathrm{B}$

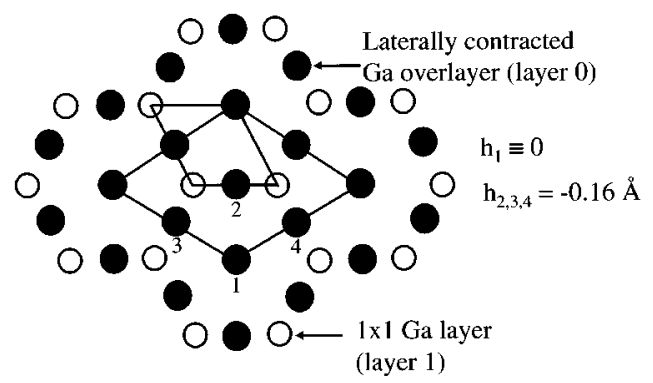

(c) registry A

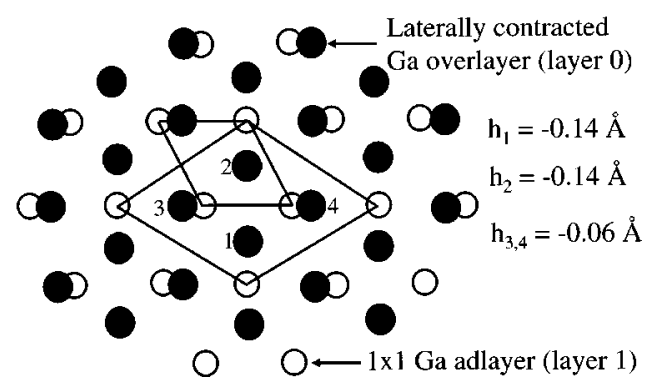

FIG. 3. (a) A schematic representation of a laterally contracted Ga bilayer above a Ga-terminated (0001) substrate. The average separations between layers are $z_{12}=2.54 \AA$ and $z_{01}=2.37 \AA$. The filled and open circles in layer 0 represent a time averaged image of the $\mathrm{Ga}$ atoms. The filled circles in layer 0 correspond to the positions at a particular time. The time averaged vertical corrugation of layer 0 is approximately $0.16 \AA$. Note: In this projection the laterally contracted monolayer (layer 0 ) has been rotated by 30 degrees for ease of viewing. (b) Projection on the $c$ plane of the top two layers for the registry $B$ structure discussed in the text. Open circles represent a $1 \times 1$ adlayer of atoms located in $T 1$ sites. (c) Projection on the $c$-plane of the top two layers for registry $A$. The heights (h) of the $\mathrm{Ga}$ atoms in layer 0 , relative to that of atom 1 in registry $B$ are listed.

in Fig. 2 is a structure consisting of a laterally contracted monolayer on top of the Ga terminated Ga-N bilayer. Relative to the ideal $1 \times 1 \mathrm{Ga}$-terminated surface such a structure contains an additional $\frac{4}{3}$ monolayer of Ga atoms. These additional atoms are located in layer 1 as shown in Fig. 2. The calculated vertical corrugation of the atoms in this layer is very small (approximately $0.03 \AA$ ) and the average vertical separation between layer 1 and layer 2 is $z_{12}=2.47 \AA$. We find that the energy of the laterally contracted monolayer structure is independent, to within $0.01 \mathrm{eV} /$ atom, of the registry of the monolayer with respect to the GaN substrate. As seen in Fig. 4, the energy of the laterally contracted mono-

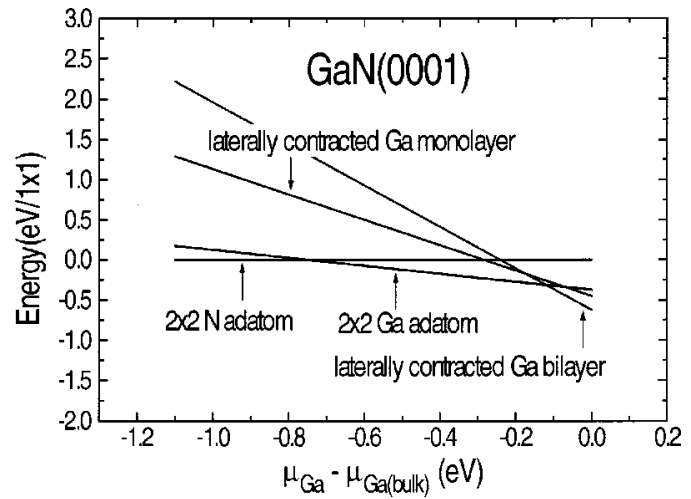

FIG. 4. Relative energies of the surfaces are plotted as a function of the Ga chemical potential. For Ga-rich conditions the most stable structure is the laterally contracted Ga bilayer.

layer structure is lower than that of the Ga $2 \times 2$ adatom model in the Ga-rich limit by $0.08 \mathrm{eV} / 1 \times 1$. We also performed calculations for structures having two layers of excess $\mathrm{Ga}$ above the GaN substrate. In the structure shown in Fig. 3(a) there is an excess of 2.33 ML of Ga atoms. Layer 1 contains 3 atoms and layer 0 contains 4 atoms in each $\sqrt{3}$ $\times \sqrt{3}$ cell. In the Ga-rich limit the energy of this structure is the lowest of any structure examined to date. In particular it is lower than the Ga adatom structure by $0.25 \mathrm{eV} / 1 \times 1$. Several inequivalent registries of layer 0 relative to layer 1 were considered and two of them are depicted in Figs. 3(b) and 3 (c). For registry $B$ the $\mathrm{Ga}$ atoms $(2,3,4)$ in layer 0 are coplanar while atom 1 is located at a height $0.16 \AA$ above these atoms. The average vertical separation between layers 0 and 1 is $z_{01}=2.37 \AA$ and the average separation between layers 1 and 2 is $z_{12}=2.54 \AA$. For registry $A$, atoms 1 and 2 are located at a height of $0.08 \AA$ below atoms 3 and 4. The average vertical separations $\left(z_{01}\right.$ and $\left.z_{12}\right)$ are almost identical to those calculated for registry $B$. Within a tolerance of about $0.02 \mathrm{eV} / 1 \times 1$ the energy of the $\sqrt{3} \times \sqrt{3}$ laterally contracted bilayer structure is independent of the registry with the substrate.

The electron diffraction data ${ }^{1}$ implies that the pseudo- 1 $\times 1$ structure is incommensurate. For computational convenience we have employed a $\sqrt{3} \times \sqrt{3}$ cell to model a laterally contracted bilayer structure. Given the insensitivity of the energy with respect to the registry, it is apparent that we could in principle form an energetically favorable incommensurate laterally contracted bilayer structure by means of a slight modification of the average in-plane Ga-Ga spacing. We therefore believe that an incommensurate laterally contracted bilayer model would be energetically favorable and consistent with the electron diffraction data.

The $1 \times 1$ corrugation seen in the STM image may arise from the dynamic behavior of the Ga atoms in layer 0 . It has been proposed ${ }^{1}$ that on the time scale of the STM scan the local registry of the atoms underneath the tip is changing rapidly. If, for a given position of the tip, we average the expected STM current over all possible registries, then a 1 $\times 1$ corrugation will be observed. The present calculations show that a $\mathrm{Ga}$ atom will be closer to the tip when it is positioned directly above one of the atoms in layer 1, e.g., in site 1 of registry $B$, and farther from the tip when it is posi- 
tioned in hollow sites, e.g., sites 1 or 2 of registry $A$. In this particular case, the difference in height of the $\mathrm{Ga}$ atom is about $0.14 \AA$.

Figure 4 shows the energies of the laterally contracted monolayer and bilayer structures together with those of the $2 \times 2 \mathrm{Ga}$ and $\mathrm{N}$ adatom structures. The $2 \times 2 \mathrm{~N}$-adatom model is energetically favorable under very $\mathrm{N}$-rich (Ga-poor) conditions, but as the chemical potential of $\mathrm{Ga}$ is increased, the $2 \times 2 \mathrm{Ga}$-adatom model becomes more favorable than the $\mathrm{N}$-adatom model. ${ }^{3-5}$ As the Ga chemical potential is increased towards its maximum possible value, structures containing more $\mathrm{Ga}$ atoms on the surface become more favorable. Until now, though, none of these Ga-rich structures were found to become more stable than the $2 \times 2 \mathrm{Ga}$-adatom model. As shown in Fig. 4, when a lateral contraction of the $\mathrm{Ga}$ adlayers is included, we obtain bilayer structures that are energetically favorable in the Ga-rich limit.

A key factor in stabilizing laterally contracted bilayer structures is the energy reduction due to the reduced $\mathrm{Ga}-\mathrm{Ga}$ spacing in the top layer. To illustrate this point we performed total energy calculations for a free-standing hexagonal bilayer of $\mathrm{Ga}$ as a function of its in-plane lattice constant. The calculations establish that a substantial reduction in energy is achieved by reducing the in-plane Ga-Ga separation from the value corresponding to a $1 \times 1$ surface $(3.17 \AA)$ to a value that is closer to a typical Ga-Ga spacing in bulk Ga $(2.7 \AA) .{ }^{15}$ As shown in Fig. 5, a reduction in surface energy ${ }^{16}$ of 25 meV/ $\AA^{2}$ is achieved by reducing the in-plane Ga-Ga spacing from $a_{1 \times 1}=3.17$ to $a_{c}=2.75 \AA$. For perspective we note that surface energies of $\mathrm{GaN}$ are typically of order $\sim 100$ meV/ $/ \AA^{2}$. Thus the contraction results in a substantial reduction in energy. It is also very important to note that the minimum in the energy occurs for a hexagonal lattice constant that is very close to $a_{c}=2.75 \AA$. This suggests that we are able to capture most of the energy benefit of the reduced Ga-Ga separation with use of the $\sqrt{3} \times \sqrt{3}$ cell. In any case, the energy shown in Fig. 4 is an upper bound on the energy of the optimal laterally contracted bilayer structure.

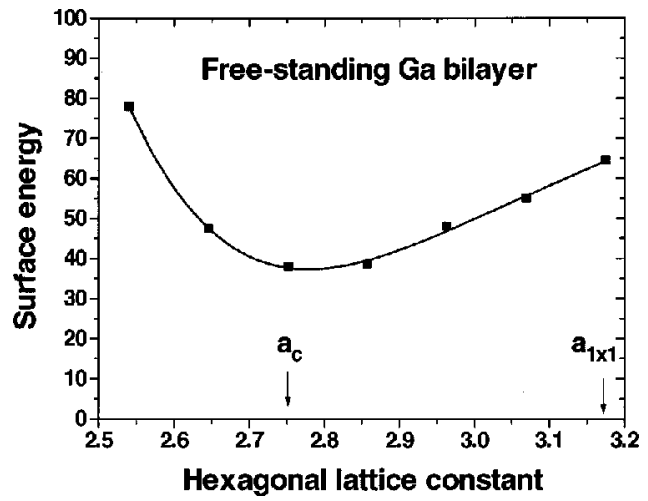

FIG. 5. The surface energy (in meV/ $\AA^{2}$ ) (Ref. 16) of a freestanding bilayer of $\mathrm{Ga}$ is plotted as a function of the in-plane hexagonal lattice constant (in $\AA$ ).

It has been determined by Hacke et al. that in the Ga-rich regime where smooth growth is observed, the activation energy for $\mathrm{Ga}$ desorption is very close to the activation energy for evaporation of metallic $\mathrm{Ga}^{2}{ }^{2}$ This result is explained naturally by the Ga bilayer model, for which one expects that the energy to remove a $\mathrm{Ga}$ atom from the top Ga layer is almost identical to that of removing an atom from bulk Ga.

In conclusion we have demonstrated that laterally contracted bilayer structures are energetically favorable under Ga-rich conditions. We have shown that the stability of these structures may be traced to the energy benefit of a reduced $\mathrm{Ga}-\mathrm{Ga}$ spacing in the surface adlayers. We have shown that the energy of these structures is insensitive to the lateral position of the adlayers, indicating that the lateral mobility of the atoms in this adlayer may be high.

This work was supported in part by the Office of Naval Research Contract Nos. N00014-99-C-0161 (Xerox) and N00014-96-1-0214 (CMU). Work in Berlin was supported by the German Science Foundation (Contract No. Ne 428/42).
${ }^{1}$ A. R. Smith, R. M. Feenstra, D. W. Greve, M. S. Shin, M. Skowronski, J. Neugebauer, and J. E. Northrup, J. Vac. Sci. Technol. B 16, 2242 (1998).

${ }^{2}$ P. Hacke, G. Feuillet, H. Okumura, and S. Yoshida, Appl. Phys. Lett. 69, 2507 (1996).

${ }^{3}$ A. R. Smith, R. M. Feenstra, D. W. Greve, J. Neugebauer, and J. E. Northrup, Phys. Rev. Lett. 79, 3934 (1997).

${ }^{4}$ K. Rapcewicz, M. B. Nardelli, and J. Bernholc, Phys. Rev. B 56, 12725 (1997).

${ }^{5}$ Qi-Kun Xue, Q. Z. Xue, R. Z. Bakhtizin, Y. Hasegawa, I. S. T. Tsong, T. Sakurai, and T. Ohno, Phys. Rev. Lett. 82, 3074 (1999).

${ }^{6}$ A. R. Smith, R. M. Feenstra, D. W. Greve, M. S. Shin, M. Skowronski, J. Neugebauer, and J. E. Northrup, Appl. Phys. Lett. 72, 2114 (1998).

${ }^{7}$ F. Widmann et al., Appl. Phys. Lett. 73, 2642 (1998).

${ }^{8}$ P. Waltereit, O. Brandt, and K. H. Ploog, Appl. Phys. Lett. 75, 2029 (1999).

${ }^{9}$ A. R. Smith, R. M. Feenstra, D. W. Greve, M. S. Shin, M. Skow- ronski, J. Neugebauer, and J. E. Northrup, Surf. Sci. 423, 70 (1999).

${ }^{10}$ V. Ramachandran et al., J. Cryst. Growth 209, 355 (2000).

${ }^{11}$ M. Bockstedte, A. Kley, J. Neugebauer, and M. Scheffler, Comput. Phys. Commun. 107, 187 (1997).

${ }^{12}$ J. E. Northrup, R. Di Felice, and J. Neugebauer, Phys. Rev. B 55, 13878 (1997).

${ }^{13}$ J. E. Northrup and J. Neugebauer, Phys. Rev. B 53, 10477 (1996).

${ }^{14}$ Guo-Xin Qian, R. M. Martin, and D. J. Chadi, Phys. Rev. B 38, 7649 (1988).

${ }^{15}$ J. Donohue, The Structure of the Elements (Robert E. Krieger Publishing Co.; Malabar, Florida; 1982). In elemental Ga each atom has seven neighbors. The average value of the Ga-Ga separation is $2.70 \AA$.

${ }^{16}$ We define the surface energy of the free standing bilayer to be equal to $1 / 2\left[E(a)-2 \mu_{(\mathrm{Ga}(\text { bulk }))}\right] / A$ where $E(a)$ is the energy per cell of the free standing $\mathrm{Ga}$ bilayer and $A=(\sqrt{3} / 2) a^{2}$ is the area of the unit cell. Each cell contains two Ga atoms aligned along the $c$ axis of the cell. 